fear of not gaining consent, due to a lack of understanding about radiation doses or not knowing how to present this information to parents/carers? We present information on radiation doses from different radiological images and propose a dose counselling model to help doctors overcome these challenges.

\section{G293(P) DOES EVERY CHILD REFERRED TO PAEDIATRIC OUT PATIENTS NEED TO BE SEEN?}

S Williamson, A Kanani, N Dlamini, R Mulik. Paediatrics, Heart of England Foundation Trust, Birmingham, UK

\subsection{6/archdischild-2015-308599.270}

Aims In the UK, the referral rate from primary care to General Paediatrics has drastically increased from from 15.5 to 25.7/ 1000 children per annum1. A Cochrane review identified that 'local educational interventions involving secondary care specialists and structured referral guidelines are the only interventions shown to negatively impact referral rates'2.

This study investigated whether written dialogue with GPs could empower them to appropriately manage potential General Paediatric out patient referrals in the community.

Method A retrospective case note review of 300 referrals which were managed with individualised advice letters rather than outpatient appointments between October 2012 and March 2014 was conducted.

Results All children under the age of 1 were offered outpatient appointments. The distribution of age groups is illustrated in Figure 1. The system based referrals are demonstrated in Table 1.

$84 \%(n=253)$ of referrals were managed by a single advice letter. Within this group, only 26\% $(n=66)$ were re directed to more appropriate services. Of the remaining 47 referrals, 38\% ( $n=18$ ) were offered outpatient appointments after further telephone discussion with the GP; 57\% $(\mathrm{n}=27)$ were re referred after the GP received the advice letter. Of the patients offered out patient appointments, 40\% ( $\mathrm{n}=19)$ were discharged after the initial consultation and $4 \%(n=2)$ did not attend. No adverse outcomes were noted.

Conclusion This study demonstrates that through supporting GP colleagues, selected outpatient referrals can be appropriately managed in the community. In our trust 253 new General Paediatric appointments would represent 41 General Paediatric clinics. However the 'Payment by Result' offers perverse financial incentive to manage more patients in secondary care. If adopted regionally this approach would improve patient care in the

\section{Distribution of Age Groups}

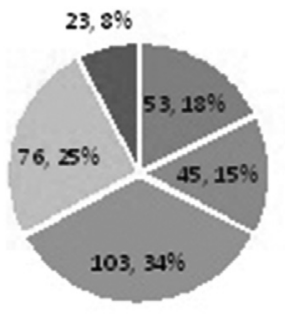

- Age $1-2$ years = Age $3-5$ months $=$ Age $6-10$ years

" Age $11-15$ years a Age $16-17$ years

Abstract G293(P) Figure 1 Distribution of age groups
Abstract G293(P) Table 1 System based referrals

\begin{tabular}{l|c|}
\multicolumn{1}{c|}{ System } & Number (\%) \\
\hline Gastrointestinal & $108(36)$ \\
Nephrology/Urology & $80(26.7)$ \\
Generally Umwell & $25(8.3)$ \\
Neurology & $22(7.3)$ \\
Abnormal Results & $19(6.3)$ \\
Musculoskeletal & $14(4.8)$ \\
Respiratory/ENT & $6(2.0)$ \\
Cardiology & $6(2.0)$ \\
Puberty & $4(1.3)$ \\
Miscellaneous & $16(5.3)$ \\
\hline
\end{tabular}

community and free up Consultant time towards consultant delivered acute care.

\section{G294(P) ONLY A QUARTER OF CHILDREN UNDER 5 YEARS HAVE EVER TAKEN VITAMIN D SUPPLEMENTS}

D Denaxa, A Colthorpe, G Bradley, A Mukherjee. General Paediatrics, Royal Oldham Hospital, Manchester, UK

\subsection{6/archdischild-2015-308599.271}

Aim It has been over a year since the $\mathrm{RCPCH}$ circulated interim guidance regarding vitamin D supplements in childhood recommending that breastfed babies from birth and formula fed infants from 6 months should receive vitamin D until 5 years old. Our aim was to assess the percentage of eligible children that take supplements and secondarily vitamin D intake in pregnant and breastfeeding women.

Methods We performed a prospective audit in children presenting to the paediatric assessment unit aged from birth up to 5 years from 20/09/14 for 2 months. We recorded: age, ethnic group, gestational age at birth, breastfeeding history, whether patients have taken or taking vitamin $\mathrm{D}$ and the indication for that. We also recorded whether the child belonged to a risk group due to prematurity, neurodisability or gastrointestinal problem and whether the child ever presented with seizures due to vitamin D deficiency.

Secondary outcomes were percentage of women taking vitamin D supplements during pregnancy and breastfeeding.

Results We recruited 158 patients with a mean age of 18 months, 57\% were males and 33\% were either Afrocarribean or Asian. $15 \%$ of the children were at high risk: $13 \%$ due to prematurity and $2 \%$ due to neurodisability. At the time of the audit only $17 \%$ of children were taking vitamin D (image 1 ) and only $25 \%$ had ever taken vitamin D. Of those that were breastfed and less than 6 months 29\% were on supplements (image 2); of those older than 6 months only 21\% (image 3). Vitamin D was started by parents in $29 \%$ and the remaining majority by health professionals. Half of the mothers took vitamin D in pregnancy and only 20\% during breastfeeding.

Conclusions Despite the circulation of the RCPCH guidelines to paediatricians only a quarter of children under five have ever been on vitamin $\mathrm{D}$. All health care professionals in primary and 
secondary care should work together towards creating clear and updated policies regarding vitamin D supplements in childhood, pregnancy and lactation, and more work on implementing these policies is needed.

\section{G295(P) PEDESTRIAN DEATHS IN IRISH CHILDREN - POTENTIAL FOR PREVENTION}

${ }^{1} \mathrm{~K}$ Hamilton, ${ }^{2} \mathrm{WL}$ Macken, ${ }^{1} \mathrm{C}$ McGarvey, ${ }^{1} \mathrm{~T}$ Matthews, ${ }^{2} \mathrm{AJ}$ Nicholson. ${ }^{1}$ National Paediatric Mortality Register, Children's University Hospital Temple Street, Dublin, Ireland; ${ }^{2}$ Department of General Paediatrics, Children's University Hospital Temple Street, Dublin, Ireland

\subsection{6/archdischild-2015-308599.272}

Aims To examine the characteristics of child pedestrian fatalities in the Republic of Ireland using the National Paediatric Mortality Register database to provide an evidence base for preventative action.

Method All child fatalities recorded on the National Paediatric Mortality Database from 1st January 2006 to 31st December 2011 were retrospectively reviewed and all road traffic related deaths were examined to identify pedestrian deaths. Passenger and cyclist deaths were excluded. Coroner's autopsy reports and death registration data were reviewed and deaths were categorised as either traffic related or non-traffic related. Deprivation scores were assigned using The Pobal Haase-Pratshke Deprivation Index.

Results There were 45 child pedestrian fatalities identified in the six year period examined. Traffic related deaths accounted for $58 \%$ vs. $42 \%$ non-traffic related. Analysis of the deaths showed there was a male preponderance. Those with a deprivation index score of "marginally below average" accounted for the majority $(53 \%)$ of deaths. There was a weekend trend and an evening and summer peak. The highest proportion of deaths occurred in the 1-4 year age group (53\%), with $28 \%$ due to low speed vehicle rollovers involving the vehicle in a reversing manoeuvre, mainly occurring in residential driveways.

Conclusion Child pedestrian fatalities are highly preventable through the modification of risk factors including behavioural, social and environmental. Most of the effort in preventing child pedestrian injuries in Ireland has focused on education of children. An area which receives little focus is non-traffic related deaths. These tragic deaths mainly involve young children/toddlers who are small in stature but independently mobile and lack the concept of personal safety. Preventative action needs to be addressed in this area.

\section{G296(P) SUPPORTING QUALITY IMPROVEMENT IN PAEDIATRICS ACROSS AN ENTIRE HEALTHCARE SYSTEM}

${ }^{1} \mathrm{~L}$ Lamb, ${ }^{1} \mathrm{G}$ Lavery, ${ }^{2} \mathrm{M}$ Smith. ${ }^{1}$ Health and Social Care Safety Forum, Public Health Agency, Lisburn, UK; ${ }^{2}$ Paediatric Department, Southern Trust, Craigavon, UK

\subsection{6/archdischild-2015-308599.273}

Aim The aim of this abstract is to describe the process of engaging and supporting those working in the paediatric service across an entire health system in a Quality Improvement (QI) programme and to describe its outcomes to date.

Methods Using the Institute for Healthcare Improvement "breakthrough collaborative" methodology, the Northern Ireland Health and Social Care Safety Forum engaged with all paediatric units to agree areas for quality improvement and to develop these into a regional driver diagram. The areas for improvement agreed upon were:

Communication - focusing on handovers, structured communications tools e.g. IPASS and SBAR, the use of safety briefings and the quality of ward rounds. The collaborative has also worked with parent representatives to develop a parent safety poster for use in all paediatric units in Northern Ireland.

Reduction in medication errors - including prescribing and administration errors

Early detection and rescue of the sick child - the collaborative have agreed a set of regional age bracketed early warning score charts and an agreed escalation protocol.

Management and Leadership - Optimising the flow of children in the acute setting

The initial three learning sessions were held over a 12 month period with action periods between. Each Trust sent a core

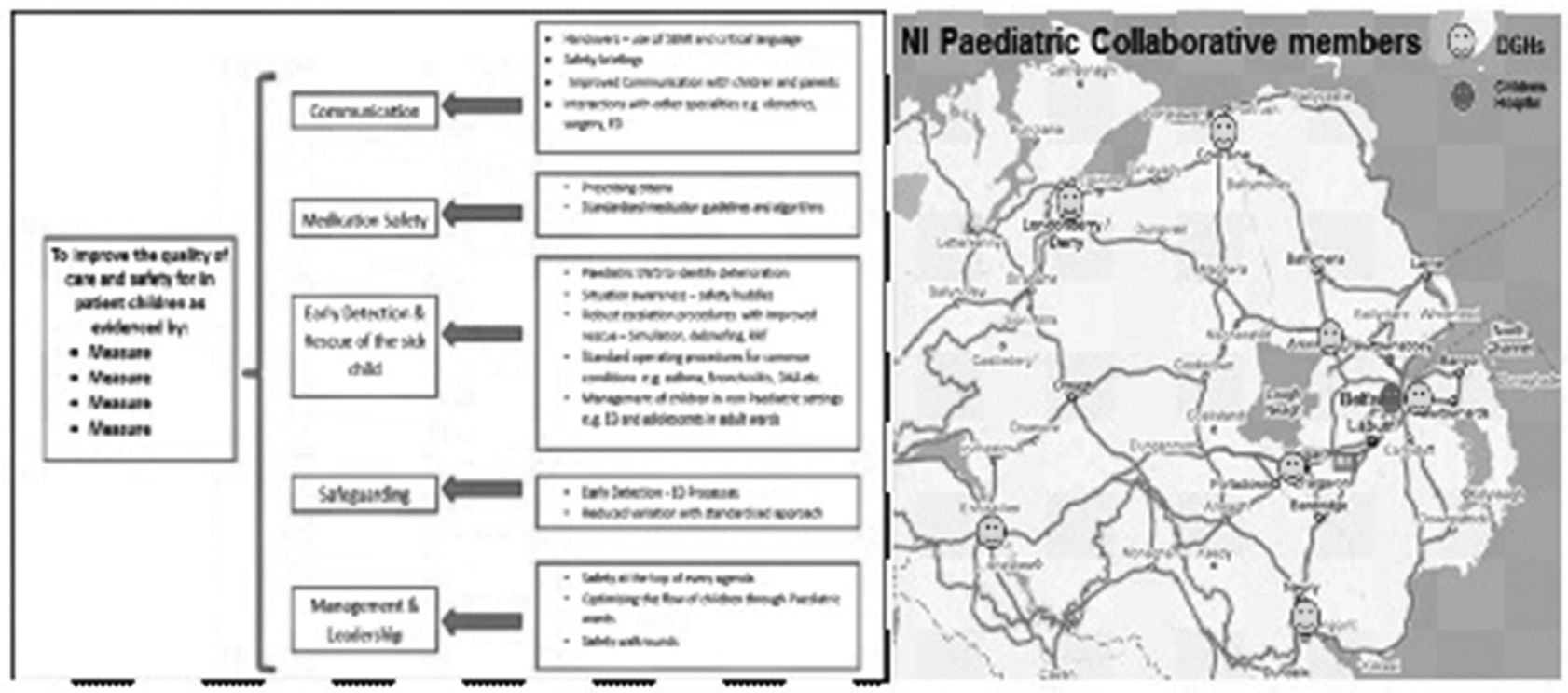

Abstract G296(P) Figure 1 Regional driver diagram and participating units 